\title{
Correction: Bustamante et al. Determining When an Algebra Is an Evolution Algebra. Mathematics 2020, 8, 1349
}

\author{
Miguel D. Bustamante $^{1} \mathbb{D}$, Pauline Mellon ${ }^{1}$ and M. Victoria Velasco ${ }^{2, *} \mathbb{D}$ \\ 1 School of Mathematics and Statistics, University College Dublin, Dublin 4, Ireland; \\ miguel.bustamante@ucd.ie (M.D.B.); pauline.mellon@ucd.ie (P.M.) \\ 2 Departamento de Análisis Matemático, Facultad de Ciencias, Universidad de Granada, 18071 Granada, Spain \\ * Correspondence: vvelasco@ugr.es; Tel.: +34-958-241-000
}

check for updates

Citation: Bustamante, M.D.; Mellon, P.; Velasco, M.V. Correction:

Bustamante et al. Determining When an Algebra Is an Evolution Algebra. Mathematics 2020, 8, 1349. Mathematics 2021, 9, 1289. https://doi.org/

$10.3390 /$ math 9111289

Received: 10 February 2021

Accepted: 15 March 2021

Published: 4 June 2021

Publisher's Note: MDPI stays neutral with regard to jurisdictional claims in published maps and institutional affiliations.

Copyright: (C) 2021 by the authors. Licensee MDPI, Basel, Switzerland. This article is an open access article distributed under the terms and conditions of the Creative Commons Attribution (CC BY) license (https:// creativecommons.org/licenses/by/ $4.0 /)$.
The authors wish to make the following corrections to this paper [1] (see corrected version in postprint [2]):

1. On page 2, paragraph 4, complete the first sentence 'In Theorem 2 we show that if $A$ is a real algebra and $B$ is a basis of $A$ then $B$ also is a basis of $A_{\mathbb{C}}$, the complexification of $A$ (with the same multiplication structure matrices) and that $A$ is an evolution algebra if, and only if, $A_{\mathbb{C}}$ is an evolution algebra' with the phrase 'and has a natural basis consisting of elements of $A^{\prime}$.

2. Replace Theorem 2 (statement and proof) with

Theorem 2. Let $A$ be a real algebra. Then $A$ is an evolution algebra if, and only if, $A_{\mathbb{C}}$ is an evolution algebra and has a natural basis consisting of elements of $A$. Moreover, if $A$ is a real evolution algebra, then every natural basis of $A$ is a natural basis of $A_{\mathbb{C}}$.

Proof. If $A$ is an evolution algebra and if $B$ is a natural basis of $A$, then obviously $B$ is a natural basis of $A_{\mathbb{C}}$. The converse direction is clear.

3. Replace Corollary 1 with

Corollary 1. Let $A$ be a real commutative algebra, let $B=\left\{e_{1}, \ldots, e_{n}\right\}$ be a basis, and let $M_{1}, \ldots$, $M_{n}$ be the m-structure matrices of $A$ with respect to $B$. Then, $A$ is an evolution algebra if, and only if, the matrices $M_{1}, \ldots, M_{n}$ (regarded as complex matrices) are simultaneously diagonalisable via congruence by means of a real matrix.

4. Add the following sentence after Corollary 1:

In [25], example 16, we give two real matrices which are diagonalisable via congruence by means of a complex matrix but not by means of any real matrix.

Finally, to aid the reader we note that reference [25] in the corrected paper [1] corresponds to reference [3] below.

The authors apologize for any inconvenience caused and state that the scientific conclusions are unaffected. The original article has been updated.

Conflicts of Interest: The authors declare no conflict of interest. 


\section{References}

1. Bustamante, M.D.; Mellon, P.; Velasco, M.V. Determining When an Algebra Is an Evolution Algebra. Mathematics 2020, 8, 1349. [CrossRef]

2. Bustamante, M.D.; Mellon, P.; Velasco, M.V. Determining When an Algebra Is an Evolution Algebra. arXiv 2021, Postprint. arXiv:2102.04493 [math.RA].

3. Bustamante, M.D.; Mellon, P.; Velasco, M.V. Solving the problem of simultaneous diagonalization of complex symmetric matrices via congruence. SIAM J. Matrix Anal. Appl. 2020, 41, 1616. [CrossRef] 